\title{
Analyse de la radioprotection des premières opérations de microbillage des tubes de générateur de vapeur des tranches $900 \mathrm{MWe}^{\star}$
}

\author{
J. LOCHARD, P. PAGĖS, F. FAGNANI**, P. HALLER ${ }^{\star \star \star}$,

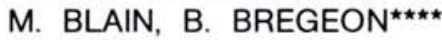 \\ (Manuscrit reçu le 12 juin 1987)
}

\begin{abstract}
RÉSUME
L'article présente les principaux enseignements, sous l'angle de la radioprotection, des premières opérations de microbillage des tubes de générateur de vapeur effectuées au cours de l'année 1986 sur les tranches 900 MWe du parc français. L'analyse dosimétrique concerne les expositions collective et individuelle, ainsi que les facteurs ayant contribué à l'évolution des doses au cours des chantiers successifs. Les principales actions concernant la mise au point des équipements, l'organisation du travail ou la mise en place de protections sont également examinées. Les résultats présentés montrent l'importance de la prise en compte de la radioprotection dès la conception et la préparation de ce type de chantier ainsi que le rôle fondamental de l'analyse du retour d'expérience.
\end{abstract}

\section{ABSTRACT}

The paper presents the main lessons, from the viewpoint of radiation protection, of the first shotpeening operations of steam generator tubes conducted in 1986 in a number of 900 MWe units of the French PWRs. Collective and individual exposures, as well as the dosimetric factors having contributed to the dose evolution of the successive operations were analysed. The main actions concerning modifications of equipments, working conditions and implementations of specific protection systems were also examined. The results presented show the importance of integrating radiation protection considerations at the design and preparation stage of this type of operation, and the fundamental role of past experience analysis.

* Cette étude a été réalisée à l'initiative du Département Sécurité Environnement Radioprotection d'Electricité de France dans le cadre d'un contrat avec le Centre d'étude sur l'évaluation de la protection dans le domaine nucléaire.

** Centre d'étude sur l'évaluation de la protection dans le domaine nucléaire (CEPN), BP 48, 92263 Fontenay-aux-Roses Cedex.

*** Electricité de France, Département Sécurité - Radioprotection - Environnement, BP 114, 93203 Saint-Denis Cedex 1.

**** Framatome, Service radioprotection, 10, rue J. Récamier, 69006 Lyon. 


\section{PRÉSENTATION}

Les premières opérations de microbillage (le principe du microbillage est présenté dans l'encadré ci-contre) des tubes de générateur de vapeur, effectuées par Framatome sur les tranches 3 des centrales de Doël (Belgique), en juillet 1985, Gravelines et Dampierre dans le courant de l'automne 1985 , se sont soldées sur le plan dosimétrique par une exposition collective totale de l'ordre de 0,9 homme.sievert pour 3 générateurs de vapeur et une exposition individuelle significative puisque le microbillage de Gravelines 3 par exemple, montrait que le quart de l'effectif étant intervenu avait reçu une dose supérieure à $10 \mathrm{mSv}$.

Dès ces premiers résultats connus, et considérant l'ampleur du programme de microbillage prévu (une vingtaine de tranches), un ensemble d'actions a été engagé pour tenter d'améliorer les résultats dosimétriques. Ces actions ont d'abord porté sur la conception du matériel de microbillage ainsi que sur sa fiabilité, et les procédures de radioprotection prévues initialement ont été perfectionnées. Une réflexion s'est également engagée, au niveau de certaines centrales ayant déjà effectué un microbillage et devant en refaire un autre, pour rechercher des moyens de réduire les expositions collectives et individuelles. Les microbillages suivants de Tricastin 3 et Bugey 2, qui sont intervenus au début de l'année 1986, ont largement bénéficié de cette mobilisation, puisque la dose collective a été ramenée aux alentours de 0,5-0,6 h.Sv. Du point de vue des expositions individuelles, le nombre d'agents ayant reçu une dose supérieure à $10 \mathrm{mSv}$ a été ramené à $15 \%$ de l'effectif. Cette tendance à la réduction des expositions s'est encore poursuivie sur les chantiers suivants : 0,4 h.Sv environ pour les opérations de Blayais 1, Dampierre 4, Bugey 5 et Tricastin 2.

L'objet de cet article est de présenter les principaux résultats d'une étude [3] effectuée sur la dosimétrie des premières opérations de microbillage afin de mettre en évidence les facteurs qui ont joué au niveau de cette évolution très favorable des doses. Cette étude qui porte sur la période allant jusqu'à mai 1986 a été réalisée à partir d'une synthèse de l'ensemble des informations recueillies par EdF et Framatome et d'une enquête auprès des centrales concernées. A l'exception de la première opération de Doël, l'analyse ne concerne que les microbillages réalisés sur les tranches du parc français de centrales. II convient de noter qu'au cours de la période étudiée, Framatome a réalisé d'autres microbillages à l'étranger, notamment en Espagne, en Belgique et en Suède.

\section{L'ANALYSE DES RÉSULTATS DOSIMÉTRIQUES}

\subsection{L'exposition collective}

\subsubsection{La dose intégrée sur le site}

La dose collective du microbillage recouvre l'ensemble des opérations effectuées sur le site, depuis l'arrivée du matériel jusqu'à son départ, y compris les travaux de préparation du chantier, en particulier le montage de sas de confinement supplémentaires sous les générateurs de vapeur pour les 


\section{LE PRINCIPE DU MICROBILLAGE}

Une des principales causes de fuites entre les circuits primaire et secondaire dans les REP est la fissuration des tubes des générateurs de vapeur dans la zone dite de "transition de dudgeonnage" soumise à des contraintes de traction (le dudgeonnage assure la fixation des tubes par gonflement dans les alésages de la plaque tubulaire).

Le microbillage est un procédé qui permet de limiter ces causes de fissures en créant un régime de contraintes de compression dans l'épaisseur des tubes. Les premiers essais ont montré que l'on pouvait retarder, voire stopper, la formation et la propagation des fissures.

Le principe en est simple et peut s'assimiler à un sablage. On projette des billes sur la peau interne des tubes au moyen d'une buse panoramique animée d'un mouvement de translation. L'outillage est positionné sous la plaque tubulaire par un porteur automatisé, type bras ou araignée. La projection est assurée par une pression d'air régulée. Ces billes sont ensuite récupérées par un système d'aspiration par pompe à vide. L'intensité du microbillage est vérifiée par la flèche que prend une plaquette de tôle calibrée fixée sur un dispositif conventionnel et dont une face est exposée au jet de microbilles (test dit de l'éprouvette ALMEN).

Les billes sont en INCONEL 600 (même matériau que les tubes) et ont un diamètre compris entre 2 et 4 dixièmes de millimètre. La pression d'injection des billes est de l'ordre de 2 bars. Le déplacement du porte-outil permet de se positionner en face de chacun des 3300 tubes à traiter par générateur de vapeur. L'introduction de la buse dans le tube est réalisée à l'aide d'un tireur-pousseur qui guide le tuyau d'amenée de billes.

équipements de microbillage. Ce travail de préparation est normalement pris en charge par une entreprise de servitudes qui assure également le nettoyage final du chantier. Quant aux opérations assurées par Framatome, outre le déchargement et le chargement des équipements sur camion, elles peuvent être regroupées selon trois postes principaux:

- l'installation du matériel dans des tentes de confinement en vinyle situées dans le bâtiment réacteur aux niveaux $4 \mathrm{~m}$ et $8 \mathrm{~m}$ (appelés dans la suite du texte "sas $4 \mathrm{~m}$ et $8 \mathrm{~m}$ ");

- le microbillage proprement dit avec les contrôles de qualité associés (tests d'Almen et de recouvrement);

- le repli du chantier (démontage-évacuation).

A ces opérations qui sont à l'origine de la quasi-totalité des doses reçues sur le site, il faut également ajouter celles reçues par le personnel de la centrale qui est amené à intervenir sur le chantier à l'occasion de mesures dosimétriques, de contrôles, ou éventuellement de difficultés techniques.

\subsubsection{L'évolution de la dose collective}

La figure 1 visualise l'évolution de l'exposition collective depuis le premier microbillage de Doël 3 . 


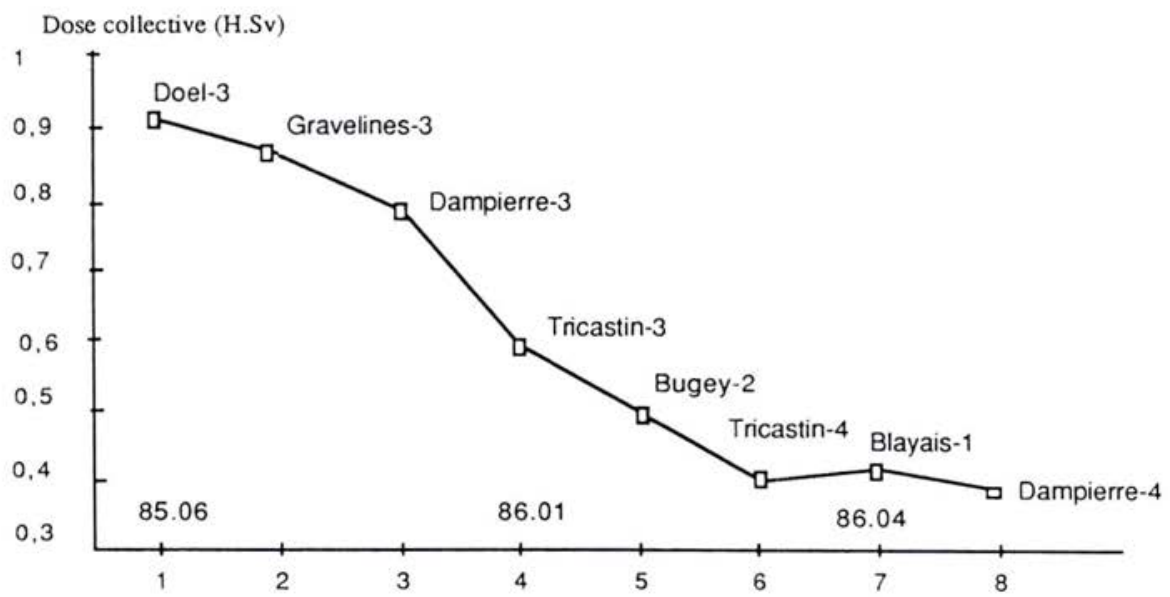

Fig. 1. - Evolution de la dose collective au cours des microbillages.

La réduction régulière du bilan dosimétrique au fil du temps traduit les bénéfices du "retour d'expérience". Si l'on considère que l'on est passé de 0,9 à 0,4 h.Sv par tranche environ, la diminution de la dose collective a donc été de l'ordre de $55 \%$.

\subsubsection{La répartition de la dose sur le site selon le type d'intervenant}

Le tableau I récapitule les doses reçues en centrale par Framatome, les entreprises chargées des servitudes et EdF. On constate que la dose Framatome représente de $85 \%$ à $88 \%$ et celle des agents EdF de 1 à $2 \%$ de la dose totale, le reste allant aux servitudes. C'est donc sur le personnel Framatome que repose l'essentiel du "poids" dosimétrique de l'opération.

\section{TABLEAU I}

Répartition de la dose collective "microbillage" entre les divers intervenants

\begin{tabular}{|l|c|c|c|c|c|c|c|}
\hline & \multicolumn{2}{|c|}{ Framatome } & \multicolumn{2}{c|}{ Servitudes } & \multicolumn{2}{c|}{ EdF } & \multirow{2}{*}{$\begin{array}{c}\text { Total } \\
\text { h.mSv }\end{array}$} \\
\cline { 2 - 7 } & h.mSv & $\%$ & h.mSv & $\%$ & h.mSv & $\%$ & \\
\cline { 2 - 7 } Gravelines 3 & 760 & 89 & 86 & 10 & 9 & 1 & 855 \\
Dampierre 3 & 677 & 85 & 105 & 13 & 18 & 2 & 800 \\
Tricastin 3 & 515 & 86 & 65 & 11 & 20 & 3 & 600 \\
Bugey 2 & 450 & 88 & $50 *$ & 10 & 11 & 2 & 511 \\
Tricastin 4** & 223 & 82 & 38 & 14 & 12 & 4 & 273 \\
Blayais 1 & 370 & 88 & 45 & 11 & 5 & 1 & 420 \\
Dampierre 4 & 337 & 86 & 52 & 13 & 5 & 1 & 394 \\
\hline * Estimation. ** Résultats pour 2 générateurs de vapeur. & & \\
\hline
\end{tabular}




\subsubsection{La dose intégrée hors du site}

Pour être exhaustif, il convient d'ajouter à la dose reçue en centrale celle relative aux opérations de décontamination et de maintenance du matériel de microbillage assurées dans l'usine B de la Cogéma à Tricastin. Pour chaque microbillage d'une tranche ( 3 générateurs de vapeur), un train d'outillage est mobilisé, soit trois jeux complets d'équipements plus un jeu en réserve (pour les dépannages d'urgence). A la fin juin 1986, 3 trains étaient en service pour assurer les opérations en France et à l'étranger.

Les données dosimétriques disponibles à l'époque de l'enquête relatives à ces opérations de décontamination-maintenance étaient assez partielles. Elles concernaient les mois de février, mars et avril 1986. Sur la base de ces résultats, on peut estimer que la dose maintenance hors centrale est de l'ordre de 50 à 60 h.mSv par tranche. Cette valeur devrait logiquement être ajoutée à la dose collective reçue en centrale si l'on veut donner une image complète de l'exposition associée au microbillage d'une tranche.

\subsubsection{La répartition de la dose collective selon les principales opérations}

Les résultats dosimétriques présentés dans les fiches d'intervention établies par Framatome ont permis d'analyser la dose totale du personnel Framatome selon les principales phases du chantier de microbillage: I'installation du matériel, les opérations normales du microbillage, les contrôles de qualité (Almen et recouvrement), le démontage, le nettoyage et l'évacuation du matériel. Outre ces opérations normales, les résultats fournissent la dose collective pouvant être attribuée aux aléas. Ces aléas concernent les pannes et incidents ayant nécessité des interventions dont la fréquence et la durée ne peuvent être prévues avec suffisamment de précision.

Le tableau II récapitule l'ensemble des valeurs des doses par opération et par tranche. On peut constater une diminution constante de la part des aléas, qui passent respectivement de 0,25 à 0,05 h.Sv environ, soit de 30 à $40 \%$ de la dose totale lors des premières opérations, aux alentours de $15 \%$ à Dampierre 4. Cette diminution des aléas permet d'expliquer environ la moitié de la réduction des doses collectives constatées plus haut.

TABLEAU ॥

Répartition de la dose collective d'une tranche selon les principales opérations (homme-mSv)

\begin{tabular}{|l|l|c|c|c|c|c|c|}
\hline Date & Tranche & Installation & Microbillage & Contrôle & Repli & Aléas & Total \\
\hline 85.06 & Doel & 127 & 324 & 99 & 103 & 275 & 928 \\
85.09 & Gravelines 3 & 98 & 263 & 71 & 123 & 205 & 760 \\
85.10 & Dampierre 3 & 59 & 213 & 68 & 49 & 273 & 672 \\
86.01 & Tricastin 3 & 74 & 109 & 90 & 102 & 158 & 534 \\
86.02 & Bugey 2 & 54 & 223 & 98 & 100 & - & 475 \\
86.04 & Blayais 1 & 56 & 89 & 34 & 90 & 87 & 357 \\
86.05 & Dampierre 4 & 53 & 111 & 37 & 83 & 41 & 324 \\
\hline
\end{tabular}

VOL. $22 \cdot \mathrm{N}^{\circ} 4$ 
Si l'on s'intéresse maintenant à chacune des phases du chantier, on retrouve cette même tendance générale à la baisse avec, cependant, la mise en évidence de particularités intéressantes. Ainsi, pour l'installation, il semble que l'on ait atteint une asymptote aux environs de 50 h.mSv par tranche. En ce qui concerne le repli, il semble que les doses des derniers chantiers (environ 80 h.mSv) puissent être encore diminuées compte tenu du résultat de 50 h.mSv obtenu à Dampierre 3. Quant au microbillage proprement dit, les gains réalisés sont considérables puisque la dose a été divisée par 3, mais il ne semble pas encore se dégager de valeur asymptote.

On peut noter que si l'on retient -le meilleur résultat pour chacune des opérations, soit 55 h.mSv pour l'installation, 90 pour le microbillage, 35 pour les contrôles et 50 pour le repli, l'objectif de 250 à 300 h.mSv par tranche apparaît réalisable. Enfin, en termes de dose totale hors aléas, les phases de microbillage et de repli fournissent la contribution majeure à la dose pour les chantiers les plus performants (Blayais 1 et Dampierre 4): elles représentent $70 \%$ de cette dose.

\subsection{Les expositions individuelles}

La dimension individuelle de l'exposition du personnel intervenant sur le chantier de microbillage est un aspect très important du problème qui peut avoir des répercussions directes sur la disponibilité des intervenants et donc indirectement sur l'exposition collective. En effet, si du fait de doses individuelles trop élevées, des opérateurs doivent être écartés des chantiers, il est nécessaire de recourir à de nouveaux travailleurs ou à des sous-traitants éventuellement moins expérimentés. On peut noter, par exemple, que lors du microbillage de Dampierre 3, environ $70 \%$ du personnel Framatome avait déjà participé à une opération (60 sur 88 personnes) alors qu'à Bugey 2 , seulement $40 \%$ du personnel avait déjà cette expérience (26 sur 67).

\subsubsection{La distribution des doses individuelles}

La figure 2 montre la répartition des doses de l'ensemble des intervenants (167 personnes) à Dampierre 3 en distinguant les agents Framatome des autres. On peut constater que $37 \%$ des doses individuelles sont inférieures à $1 \mathrm{mSv}, 55 \%$ sont comprises entre 1 et $5 \mathrm{mSv}$ et $8 \%$ supérieures à $10 \mathrm{mSv}$. Aucune dose individuelle supérieure à $5 \mathrm{mSv}$ ne concerne les agents des entreprises chargées des servitudes ou d'EdF. La dose maximale est comprise entre 19 et $20 \mathrm{mSv}$. Une analyse de la répartition des doses individuelles comptabilisées au niveau de chacun des "sas" montre que c'est dans le "sas $8 \mathrm{~m}$ ", où les opérateurs interviennent sur le matériel irradiant ou dans les boîtes à eau des générateurs de vapeur que l'on trouve les doses les plus importantes. 


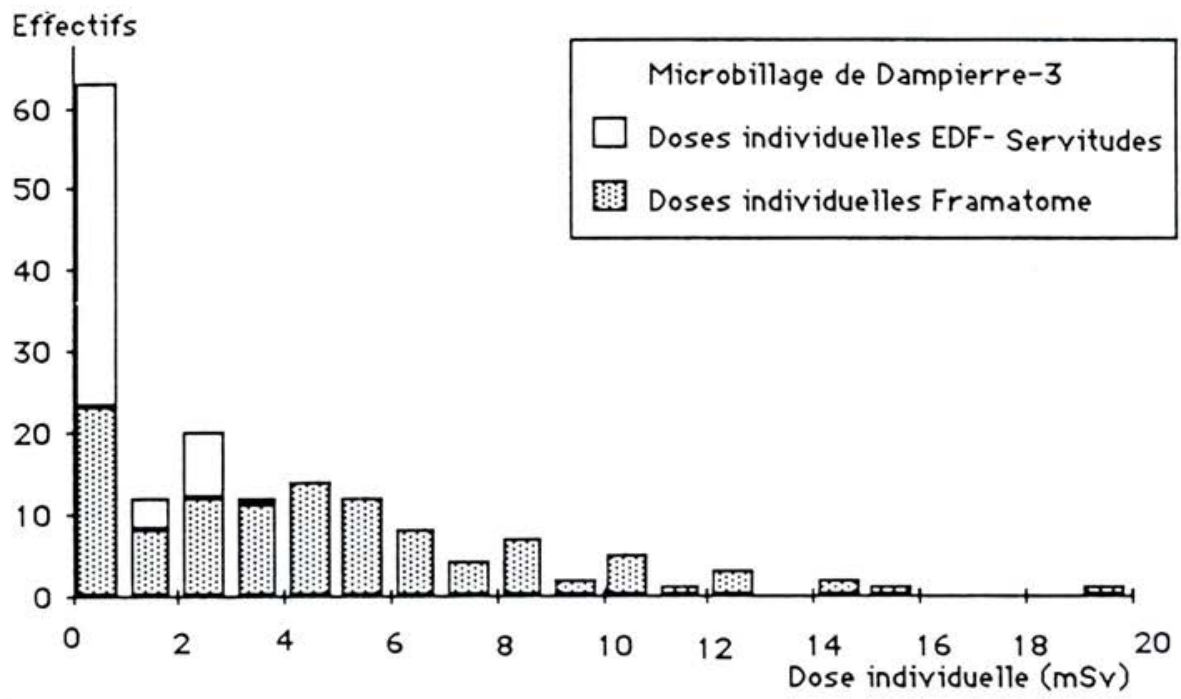

Fig. 2. - Distribution des doses individuelles lors du microbillage à Dampierre 3.

La comparaison des doses individuelles moyennes selon les postes de travail dans les différents sas (tableau III) montre que les agents Framatome reçoivent $4,24 \mathrm{mSv}$ dans le "sas $8 \mathrm{~m}$ ". contre 1,55 en moyenne dans le "sas $4 \mathrm{~m}$ ". En ce qui concerne les entreprises de servitudes, c'est, par contre, au niveau du "sas $4 \mathrm{~m}$ " que les doses sont les plus importantes: $1,03 \mathrm{mSv}$ contre 0,46 dans le "sas $8 \mathrm{~m}$ ".

TABLEAU III Doses individuelles moyennes dans les sas $4 \mathrm{~m}$ et $8 \mathrm{~m}$ lors
du microbillage à Dampierre 3

\begin{tabular}{|l|r|c|c|c|c|c|}
\hline & \multicolumn{2}{|c|}{ Effectif } & \multicolumn{2}{c|}{$\begin{array}{c}\text { Dose collective } \\
\text { (h.mSv) }\end{array}$} & \multicolumn{2}{c|}{$\begin{array}{c}\text { Dose moyenne } \\
(\mathrm{mSv})\end{array}$} \\
& sas $4 \mathrm{~m}$ & sas $8 \mathrm{~m}$ & sas $4 \mathrm{~m}$ & sas $8 \mathrm{~m}$ & sas $4 \mathrm{~m}$ & sas $8 \mathrm{~m}$ \\
\cline { 2 - 8 } & 89 & 96 & 138 & 408 & 1,55 & 4,24 \\
Framatome & 25 & 18 & 26 & 8 & 1,03 & 0,46 \\
Servitudes & 7 & 19 & 2 & 5 & 0,31 & 0,26 \\
EdF & & & & &
\end{tabular}

\subsubsection{Evolution de la distribution}

La diminution générale de l'exposition collective constatée se traduit par une évolution corrélative de la distribution des doses individuelles. Le phénomène est illustré par la figure 3 et le tableau IV qui montrent l'évolution de la dose moyenne et de la distribution des doses individuelles des agents Framatome lors des interventions de Gravelines 3, Bugey 2 et Blayais 1. 
La dose individuelle moyenne est passée de 8,10 à $5,60 \mathrm{mSv}$ par agent. Cette diminution a d'abord porté sur les doses supérieures à $10 \mathrm{mSv}$, les doses inférieures à $5 \mathrm{mSv}$ restant stables. Les résultats de Blayais $1 \mathrm{sem}$ blent indiquer que la diminution des doses comprises entre 5 et $10 \mathrm{mSv}$ est engagée. Globalement, le pourcentage de l'effectif ayant reçu une dose supérieure à $10 \mathrm{mSv}$ est passé de $24 \%$ à $6 \%$. Ce dernier chiffre est confirmé par celui obtenu à Dampierre 4 qui est de $5 \%$.

Ces résultats démontrent que les mesures prises pour diminuer l'exposition collective se traduisent par une diminution parallèle des doses individuelles les plus fortes. On peut estimer qu'une dose collective totale n'excédant pas 0,3 h.Sv devrait conduire à la suppression des doses supérieures à $10 \mathrm{mSv}$ et la réduction du nombre d'agents recevant des doses comprises entre 5 et $10 \mathrm{mSv}$.

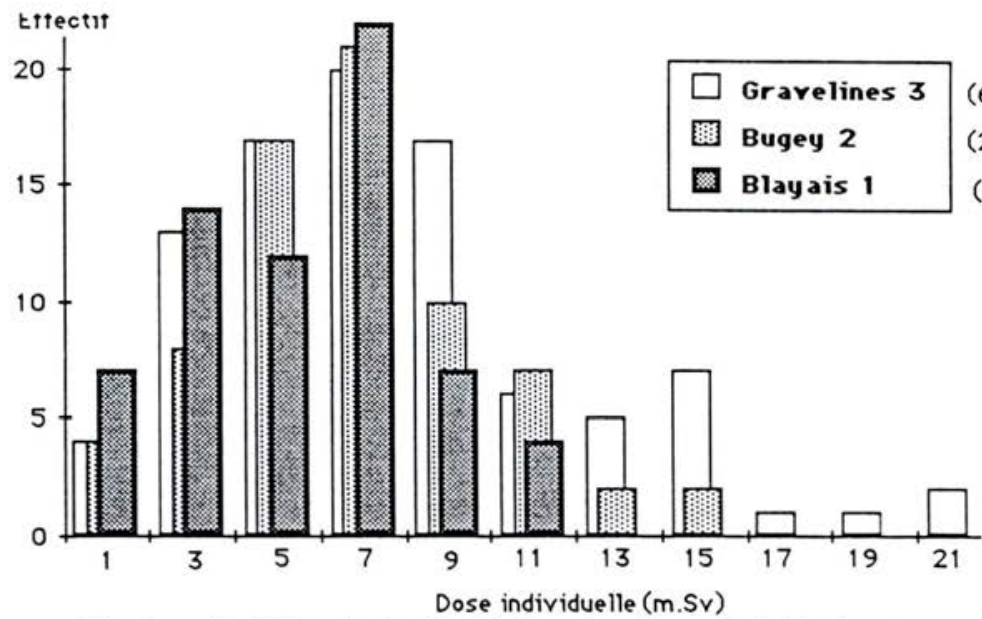

Fig. 3. - Evolution de la distribution des doses individuelles au cours de la campagne de microbillage.

TABLEAU IV

Evolution de la dosimétrie individuelle des agents Framatome au cours de la campagne de microbillage

\begin{tabular}{|l|c|c|c|}
\hline & $\begin{array}{c}\text { Gravelines } \\
\text { (septembre } \\
85 \text { ) }\end{array}$ & $\begin{array}{c}\text { Bugey 2 } \\
\text { (février 86) }\end{array}$ & $\begin{array}{c}\text { Blayais 1 } \\
\text { (avril 86) }\end{array}$ \\
\cline { 2 - 4 } $\begin{array}{l}\text { Nombre d'agents } \\
\text { Dose totale (h.Sv) }\end{array}$ & 94 & 71 & 66 \\
Dose moyenne/agent (mSv) & 0,76 & 0,47 & 0,37 \\
Répartition de la dose totale (\%) & 8,10 & 6,60 & 5,60 \\
5 5 mSv & 27 & & \\
$5-10 \mathrm{mSv}$ & 49 & 27 & 41 \\
$10-20 \mathrm{mSv}$ & 24 & 15 & 53 \\
& & & 6 \\
\hline
\end{tabular}




\subsection{L'influence du débit de dose et de la durée du travail}

L'idée généralement avancée est que l'exposition sur un chantier dépend essentiellement des débits de dose ambiants, et qu'une opération sera donc d'autant plus pénalisante sur le plan dosimétrique que les sources sont importantes. En fait, l'exposition collective est la résultante de nombreux paramètres dont le débit n'est qu'un des aspects. Ces paramètres peuvent être regroupés selon trois facteurs principaux: le niveau général des débits de dose ambiants (donc les sources), la durée effective du travail et l'effectif exposé.

Les figures 4 et 5 montrent l'évolution, pour les différentes tranches, de la dose collective moyenne par générateur de vapeur en fonction d'une part du débit de dose ambiant moyen dans les boîtes à eau des générateurs (côté chaud) et, d'autre part, de la durée moyenne de l'opération de microbillage par générateur de vapeur.

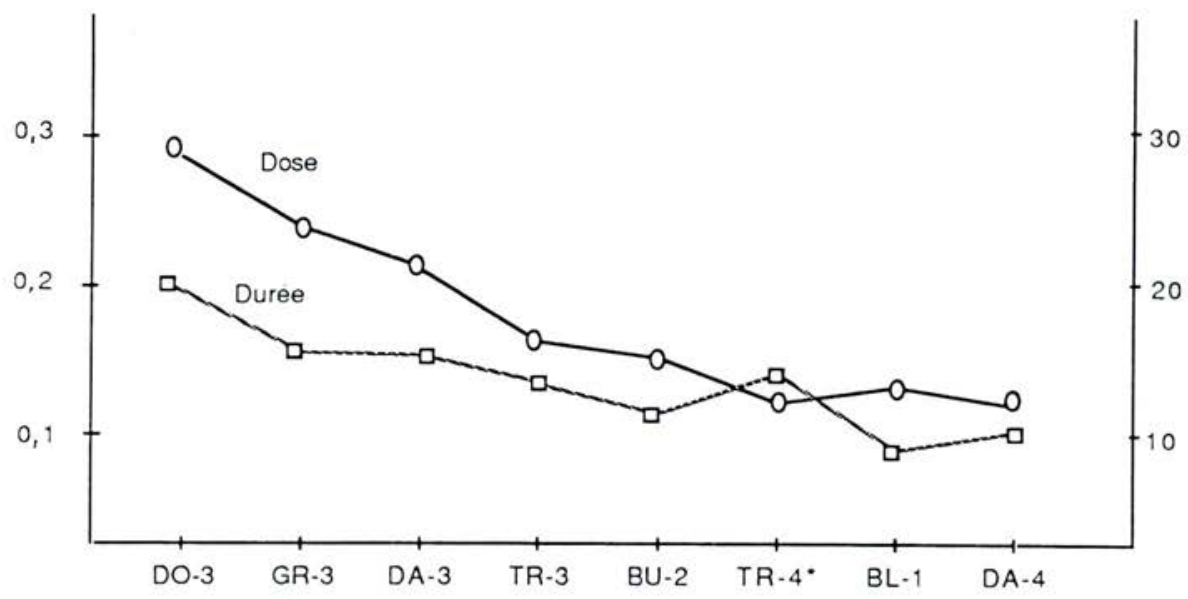

Fig. 4. - Evolution de la dose collective et de la durée du microbillage au cours de la campagne.

* Moyenne sur 2 générateurs de vapeur.

L'examen de ces graphiques suggère a priori une liaison forte entre la durée du chantier et la dose collective, ainsi qu'une relative indépendance entre le débit de dose ambiant dans la boîte à eau des générateurs de vapeur et la dose collective. Ainsi, les débits de dose importants notés à Bugey 2 n'ont pas empêché la dose collective de diminuer du fait des gains obtenus sur le plan de la durée. II faut, cependant, noter que le phénomène est particulièrement net pour les premiers chantiers, y compris celui de Bugey 2, et qu'ensuite l'influence du débit de dose semble reprendre de l'importance au même titre que celle de la durée. Ainsi, pour Tricastin 4 et Dampierre 4, malgré une augmentation assez sensible de la durée, la dose collective a continué à décroître en raison du faible débit de dose au niveau des générateurs de vapeur. 


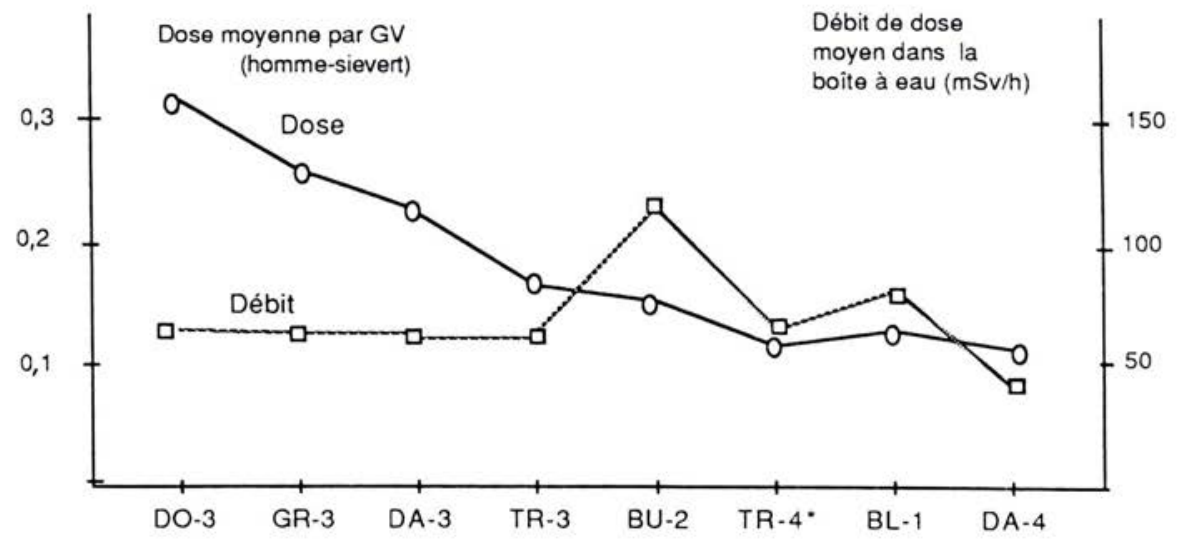

Fig. 5. - Evolution de la dose collective et niveaux des débits de dose dans les générateurs de vapeur au cours de la campagne de microbillage. * Moyenne sur 2 générateurs de vapeur.

Ces résultats montrent que la diminution rapide de la dose collective est, au départ, une conséquence presque exclusive de la réduction de la durée de l'opération. L'influence (positive ou négative) du niveau des débits de dose est négligeable par rapport aux autres facteurs de variation. Les opérations de Tricastin 4 et Dampierre 4 sont les seules pour lesquelles le rôle du débit de dose est manifeste: malgré des durées plus longues que pour les opérations précédentes, la dose a continué à décroître du fait de débits de dose particulièrement favorables. En fait, le phénomène dû à la réduction de la durée tend progressivement à atteindre ses limites (quasi-suppression des aléas, bénéfice maximal du facteur apprentissage), et l'on entre alors dans une phase où l'influence des conditions ambiantes devient un élément plus actif pour expliquer l'évolution des expositions.

Ces résultats constatés à propos du microbillage relèvent, en fait, d'un phénomène général. Des travaux antérieurs portant sur l'analyse de l'évolution des doses collectives associées aux opérations de maintenance normale dans certains réacteurs (Ringhals en Suède, Loviisa en Finlande) ont déjà suggéré le rôle dominant du facteur réduction du "temps de travail" par rapport à celui de la réduction des débits pour expliquer la diminution des doses relatives à des chantiers répétitifs [1, 2].

Sur le plan pratique, ces conclusions suggèrent que les efforts pour réduire les doses doivent en priorité porter sur les durées d'intervention plutôt que sur les mesures du type protection, tant que les opérations ne s'effectuent pas à l'optimum du point de vue de leur durée. II faut également noter que la réduction de la durée des opérations jointe à l'amélioration des 
procédures influe directement sur l'effectif nécessaire pour réaliser les opérations. Les données Framatome montrent que, depuis le début du programme de microbillage, l'effectif a été réduit d'environ $30 \%$.

\section{LES ACTIONS DE RÉDUCTION DE L'EXPOSITION}

Les gains dosimétriques observés au fil des opérations ont été obtenus d'une part par une diminution importante des aléas et, d'autre part, par l'adoption successive de diverses mesures de protection. Ces éléments résultent de la mise au point du matériel, de l'entraînement des équipes, et du retour d'expérience en général. L'examen des fiches d'intervention établies après chaque microbillage par Framatome est, à ce propos, tout à fait révélateur de la prise en compte progressive, de la part des responsables de chantier, des points faibles sur lesquels il convenait d'agir.

Lors des premiers microbillages (Doel 3, Gravelines 3), l'accent était mis essentiellement sur la nécessité d'améliorer la fiabilité et la mise au point du matériel pour faire diminuer les aléas. L'attention était également attirée sur le recyclage manuel des billes, coûteux en dose puisque les opérateurs devaient manipuler dans des conditions difficiles (tenue étanche) des pots de billes contaminées et relativement lourds. Par ailleurs, les points critiques concernant les débits de dose sur le matériel avaient été repérés au cours des cartographies effectuées dans les "sas $4 \mathrm{~m}$ et $8 \mathrm{~m}$ ". Le problème général de l'organisation du chantier était aussi mis en avant, en particulier le positionnement des équipements, afin d'éviter les zones où les débits ambiants étaient les plus pénalisants. Les opérateurs se sont rendus compte que la conception des matériels était telle qu'il était parfois très difficile de les placer dans les lieux les plus judicieux comme, par exemple, le tireur pousseur dans le "sas $8 \mathrm{~m}$ " qui devait être placé en face du trou d'homme du générateur de vapeur.

Au cours des chantiers suivants de Dampierre 3 et de Tricastin 4, qui bénéficiaient de la mise en service du recyclage automatique et qui enregistraient une nette diminution des aléas, l'attention restait encore largement fixée sur l'adaptation du matériel aux conditions de travail en zone. La mise en place de protections biologiques autour de certains équipements, voire leur intégration sur le générateur de billes ou le tireur pousseur par exemple, est également un souci qui est apparu.

L'analyse des fiches d'intervention pour les microbillages de Bugey 2, Tricastin 4, Blayais 1 et Dampierre 4 montre que les opérateurs ont continué à mettre l'accent sur certaines modifications du matériel afin de faciliter le travail (accès aux filtres, accrochage rapide des protections biologiques sur des armatures spéciales, plombage de certains composants...) mais surtout sur des problèmes d'organisation: notamment la coordination avec les centrales au sujet de la planification des replis de chantier (problèmes de conditionnement du matériel, de sa décontamination, possibilité d'utiliser des équipes de repli spécialisées...).

Ce bref aperçu de l'évolution des remarques consignées dans les fiches d'intervention, bien que non exhaustif, permet, néanmoins, de se faire une idée assez juste des questions qui sont apparues au fur et à mesure que VOL. $22 \cdot \mathrm{N}^{\circ}{ }_{4}$ 
l'expérience s'accumulait sur les chantiers. En résumé, c'est, tout d'abord, la nécessité de mieux préparer les intervenants et d'améliorer la fiabilité du matériel qui apparaît. Viennent ensuite les questions relatives à l'adaptation du matériel et de l'organisation du travail aux conditions particulières de travail en zơne irradiée et contaminée. Enfin, ce sont des problèmes de coordination avec la centrale et de planification des opérations les plus délicates qui finissent par devenir prépondérants.

L'analyse de l'évolution des chantiers montre, également, que les opérateurs ont su rapidement repérer les points critiques, en particulier ceux concernant les débits de dose, par le recours à des cartographies systématiques et répétées.

\subsection{Les actions réalisées}

Les premières modifications ont surtout porté sur l'équipement afin d'en améliorer la fiabilité, de réduire la consommation des billes et de réduire la durée de l'opération. Bien entendu, ces modifications ont eu une répercussion directe sur la dosimétrie, en particulier le recyclage automatique qui a permis un gain d'environ 20 à 30 h.mSv par générateur de vapeur. La priorité a été donnée d'abord à la mise au point du matériel et à son utilisation optimale, afin, notamment, de réduire les aléas avant les prises en compte des demandes concernant plus spécifiquement les aspects radioprotection.

A ces mesures portant sur le matériel, il convient également d'ajouter l'ensemble des mesures moins visibles qui, progressivement, ont été introduites par les opérateurs et qui concernent l'organisation du chantier et le déroulement des opérations. Au cours des diverses opérations s'est, en effet, accumulé un savoir-faire en partie consigné dans la procédure de radioprotection, mais surtout transmis directement entre les responsables sur le terrain. Ces mesures recouvrent un large éventail de "trouvailles" allant du choix de la fréquence de changement des filtres à la procédure de "rinçage" des équipements en fin de microbillage pour récupérer les billes et décontaminer au maximum le matériel avant son démontage, en passant par la disposition la plus judicieuse des divers éléments. II s'agit là de l'ensemble des éléments que l'on peut rassembler sous le terme générique de "facteur d'apprentissage". L'évolution de la dose collective associée à l'installation du matériel - qui est passée progressivement de 130 à 50 homme.mSv - semble résulter typiquement de ce type de facteur. C'est, en fait, l'ensemble de ces modifications de détail, ajoutées à celles plus spectaculaires mentionnées plus haut, qui ont permis d'obtenir des résultats globalement importants.

\subsection{L'amélioration de la productivité}

Les modifications introduites au cours des chantiers successifs ont conduit à une forte amélioration de la productivité. En ce qui concerne les éléments connus, les heures de travail effectuées par Framatome ont diminué de plus de $40 \%$, passant de $14000 \mathrm{~h}$ au niveau des premiers chantiers (Gravelines 3 et Dampierre 3) à $9000 \mathrm{~h}$ environ pour Blayais 1. De même, la consommation de billes est passée dans le même temps de $1 \mathrm{t}$ environ à 0,3 t. 
On peut estimer que, sur le plan financier, cette évolution s'est traduite par une réduction de l'ordre de $25 \%$ du coût total du chantier. Ce résultat ne prend pas en compte l'économie réalisée sur la formation et l'entraînement du personnel Framatome du fait de la diminution de la part du renouvellement du personnel due à la réduction générale du niveau des doses individuelles. Par ailleurs, à la diminution du coût direct du microbillage, il faut ajouter celle correspondante au coût de l'arrêt de tranche: le chantier n'étant plus sur le chemin critique du fait de la réduction de sa durée.

Ces quelques éléments économiques démontrent que les mesures prises dès les premiers chantiers pour remédier aux aléas et améliorer le déroulement des opérations, ont eu non seulement un effet bénéfique sur la dosimétrie collective et individuelle, mais ont également contribué à une diminution importante de son coût global.

\section{CONCLUSION}

Les résultats présentés démontrent l'efficacité des actions qui ont été entreprises aux niveaux de la fiabilité des équipements, de l'optimisation des temps d'opération et du renforcement des protections. Cette évolution très favorable ne doit pas masquer, cependant, qu'il a fallu presque une dizaine d'opérations pour obtenir ces résultats, et l'on peut penser que les enseignements tirés de ces interventions permettront, pour des interventions futures de même niveau technologique, d'améliorer la situation initiale par une prise en compte affinée des considérations radiologiques, tant au niveau de la conception générale des matériels que de la mise en œuvre de l'intervention et des mesures d'accompagnement qui s'y rapportent.

\section{REMERCIEMENTS}

Les auteurs remercient toutes les personnes qui, dans les centrales ou dans les services centraux d'EdF et de Framatome, ont collaboré directement ou indirectement à ce travail.

\section{RÉFÉRENCES}

[1] EGNER K. Cost-effectiveness of dose reduction modifications at Ringhals 2. In: Proceedings of an International workshop on Historic dose experience and dose reduction (ALARA) at nuclear power plants, Upton, May 29 - June 1, 1984. NUREG/CP-0066, 1985.

[2] LOCHARD J., PAGES P. Une analyse de l'exposition professionnelle dans les réacteurs à eau préssurisèe. In : Radiation-risk-protection, Proceedings of the 6 . International Congress of IRPA, Berlin West, 7-12 May, 1984 (Kaul A., Neider R., Pensko J., Eds). Koln: TUV Rheinland, 1984, vol. 2, 649 a - 649 d.

[3] LOCHARD J., PAGES P., FAGNANI F. Analyse de la radioprotection des premières opérations de microbillage des tubes de générateurs de vapeur des tranches 900 MWe. Rapport CEPN no 98, 1986. 